\title{
Laser-induced ultrasound transmitters for 3D photoacoustic and ultrasound tomography
}

Damien Gasteau, David Thompson, Srirang Manohar

Damien Gasteau, David Thompson, Srirang Manohar, "Laser-induced ultrasound transmitters for 3D photoacoustic and ultrasound tomography," Proc. SPIE 11077, Opto-Acoustic Methods and Applications in Biophotonics IV, 1107716 (19 July 2019); doi: 10.1117/12.2527011 


\title{
Laser-induced ultrasound transmitters for 3D photoacoustic and ultrasound tomography
}

\author{
Damien Gasteau ${ }^{\mathrm{a}}$, David Thompson ${ }^{\mathrm{a}}$, and Srirang Manohar ${ }^{\mathrm{a}}$ \\ aBiomedical Photonic Imaging group, Technical Medical Centre, University of Twente, \\ 7522NB Enschede, The Netherlands
}

\begin{abstract}
We present a 3D photoacoustic and ultrasound tomographic system intended for imaging of breast phantoms with the capacity to detect millimetric objects. The speciality of the ultrasound imaging part is that transmitters based on laser-induce ultrasound (LIUS) are used for acoustic generation. We describe the design and development of transmitters including an absorbing layer for photoacoustic generation.
\end{abstract}

Keywords: Photoacoustic imaging, Laser-induced ultrasound, PDMS, Carbon black

\section{INTRODUCTION}

The use of photoacoustic methods for breast imaging purposes is in continuous development. ${ }^{1}$ The photoacoustic effect can be exploited to create an ultrasound source specifically designed for ultrasound imaging. One alternative to a conventional piezoelectric based transducer probe is to use an absorbing material which when illuminated by a pulsed light source, produces acoustic waves via the photoacoustic effect. An efficient approach is to use an elastomer matrix in which light-absorbing particles are suspended. ${ }^{2}$ This method of ultrasound generation is named Laser Induced UltraSound (LIUS) to make a distinction with the US produced within inside the tissue by the photoacoustic effect. This approach is still in its early developments but Kruizinga et. al. ${ }^{3}$ have already shown the first examples of LIUS imaging applied to a biomedically relevant phantom. LIUS transmitters were also recently compared with conventional ultrasound imaging giving equivalent results in terms of imaging contrast in a plane wave configuration. ${ }^{4}$

In this work we propose a specific LIUS transmitter optimized for implementation in a newly developed multimodal (PA \& US) 3D tomographic setup. It is aimed to be exploited as acoustic source for imaging and speed of sound evaluation. In order to create a LIUS transmitter two elements are needed: a light source and a light absorbing material. One of the main challenges is to obtain a low frequency acoustic pulse with a reduced size LIUS transmitter by optimizing the thickness, absorption of the absorbing layer and physical properties of the materials used.

\section{MATERIALS AND METHODS}

\subsection{LIUS transmitters}

The use of a LIUS transmitter for ultrasound imaging and speed of sound evaluation implies several specific requirements to be fulfilled. The acquisition of acoustic signals for both PA and US is made using conventional detectors (Imasonics, Besanon, France) centered at $1 \mathrm{MHz}$ for optimal detection of objects of 1-2 mm in size. The LIUS transmitters must match with this bandwith and present an acoustic beam with a half angle of 20 degrees (defined at $1 / e$ ) for ideal coupling with the transducers directivity. This large opening angle has the advantage to allow insonification of a wide volume of the studied sample with a single element. The generated pressure levels also need to be sufficient for transmission through breast tissue. The LIUS transmitters are to be used in an PA and US clinical setup, the dimensions are constrained to a centimeter in diameter due to the high density of elements we wish to implement. Ideally the LIUS transmitters simple and affordable to produce but still reliable to perform well during several measurement sessions without loss of signal.

Correspondence d.b.gasteau@utwente.nl

Opto-Acoustic Methods and Applications in Biophotonics IV, edited by Vasilis Ntziachristos,

Roger Zemp, Proc. of SPIE-OSA Vol. 11077, 1107716 · (C) 2019 SPIE-OSA

CCC code: $1605-7422 / 19 / \$ 21 \cdot$ doi: $10.1117 / 12.2527011$

Proc. of SPIE-OSA Vol. 11077 1107716-1 


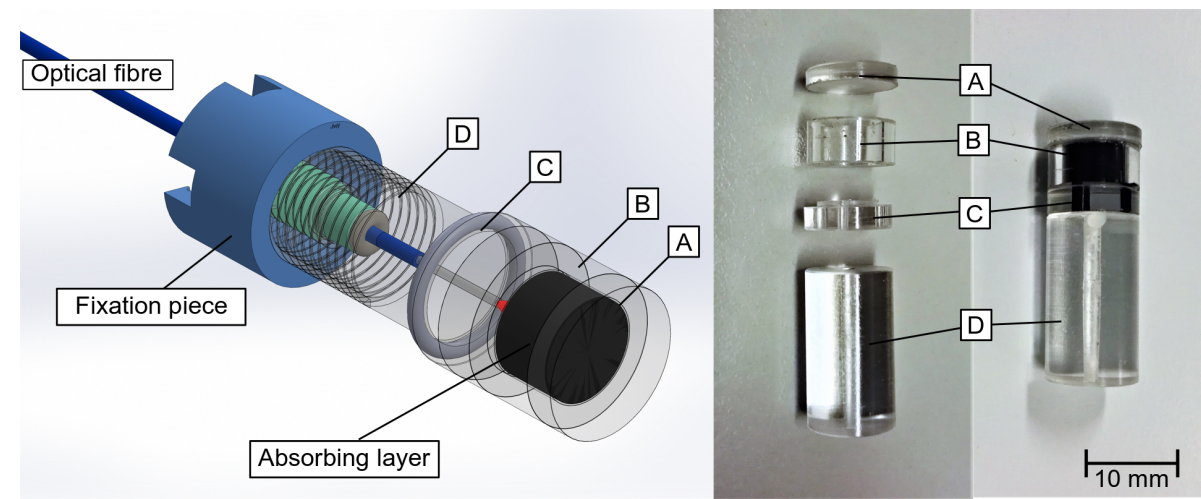

Figure 1. Design of the final LIUS transmitter (left), separated pieces (center) and mounted prototype (right). Parts A, B, C and D are composed of PMMA. PDMS mixed with Carbon Black is embedded in the part B for photoacoustic generation

One design of such a transmitter is presented in Fig 1. The main body of the transmitter (A, B, C and D) is made of a PMMA structure with a cavity (B) filled with light absorbing material. PMMA was chosen as it is easy to machine and relatively transparent (around 90 percent transmission) in the visible to NIR ${ }^{5}$ range so light can be sent through it order to achieve photoacoustic generation. An optical fibre is mounted by the back of the element illuminating the absorbing material through a PMMA layer $(\mathrm{C})$. The back part is composed a fixation piece which when assembled with the part (D) grant an easy and robust mounting on the arms of the setup. Along the main axis of the transmitter, the acoustic waves must transmit through a multilayered medium to reach the imaging domain.

For optimal generation of an acoustic pulse centred at $1 \mathrm{MHz}$, simulation using k-wave and experimental validation lead to an absorbing layer composed of PDMS prepared in a $10: 1$ ratio with curing agent and doped with $\mathrm{CB}$ at $0.13 \%$ concentration in mass to obtain an absorption coefficient of $8 \mathrm{~mm}^{-1}$. PDMS makes an ideal candidate for our application as it offers the possibility to be cast and cured in a wide range of shapes, presents high thermal expansion coefficient, and is relatively easy to mix with absorbing particles. Approximating the acoustic beam as a Gaussian beam, it is possible to predict the behaviour of the acoustic pulses propagating in the LIUS transmitter towards the imaging domain. Knowing the physical properties of the different materials composing the transmitter, the dimensions of the layers were designed to achieve optimal transmission and geometrical properties of the acoustic beam such as opening angle but also to reduce internal reflections.

\section{$2.23 \mathrm{D}$ tomography imager}



Figure 2. Imager design (left) and mounted imager with one arm and illumination fibres for PA (right)

With the goal to achieve 3D photoacoustic and ultrasound imaging of phantoms the tomographic set up presented in Fig 2 was developed. It is composed of two 3D printed arms visible on Fig 3 placed in a water tank 
in which the transducers (Imasonics) and LIUS elements are mounted. The curved side of the arms allows to have all the elements on a section of a hemisphere with $25 \mathrm{~cm}$ of diameter. The back of the arms is hollow in order to fit most of the electronic components related to the acquisition chain and fixation of the elements.
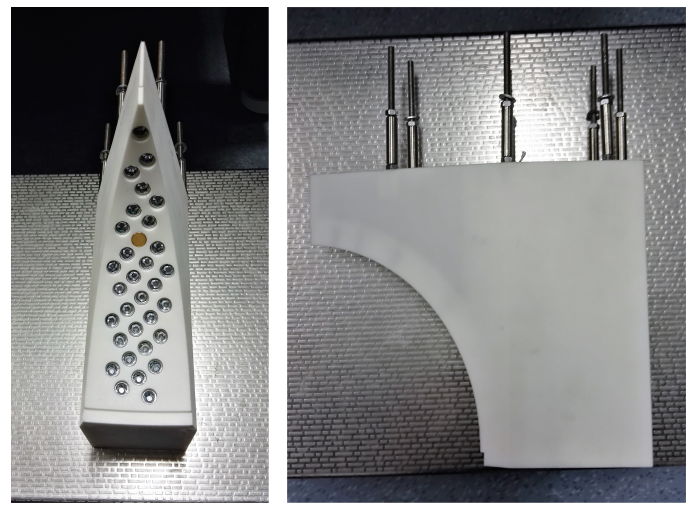

Figure 3. Curved side of the printed arm with holes for LIUS transmitter and transducer mounting (left) and side view of the arm (right)

The two arms are mounted on independent rotation stages which allows an almost complete coverage of the sample placed in the middle. Each arm is pierced by several holes arranged as a section of a non-periodical spiral in order to avoid repetitive data during scanning measurements. The density of holes is recreating a hemispherical bowl possessing 600 elements mounted on it. In consequence with the two independent arms, an important number of combinations of transmitter/detector can potentially be achieved, mostly limited by the scanning step of the stages. Only a small blind angle created by the fact that the two arms can not overlap is to be considered. In addition, 9 optical fibres for photoacoustic excitation are mounted to achieve a homogeneous illumination of the sample place in the imager. Using this multimodal set up, combined 3D PA and US images of breast phantom can thus be obtained.

\section{CONCLUSION}

We report on the development of LIUS transmitters for US imaging of breast phantoms. We described the steps leading to the final design and the fabrication process. Acoustic behaviour is predicted by numerical simulations and validated experimentally. Future LIUS transmitter will be optimized for higher energy conversion. The optical properties of the absorbing layer will adjusted for more control on the temporal and spatial acoustic characteristics.

\section{REFERENCES}

[1] Manohar, S. and Dantuma, M., "Current and future trends in photoacoustic breast imaging," (To be published).

[2] Baac, H. W., Ok, J. G., Park, H. J., Ling, T., Chen, S.-L., Hart, A. J., and Guo, L. J., "Carbon nanotube composite optoacoustic transmitters for strong and high frequency ultrasound generation," Applied Physics Letters 97(23), 234104 (2010).

[3] Kruizinga, P., Cox, B., de Jong, N., Beard, P., van der Steen, A. F., and van Soest, G., "Plane wave ultrasound imaging with a broadband photoacoustic source," in [Ultrasonics Symposium (IUS), 2012 IEEE International], 1414-1416, IEEE (2012).

[4] Thompson, D., Gasteau, D., and Manohar, S., "Spatially compounded plane wave imaging using a laser induced ultrasound source," (To be published).

[5] Prajzler, V., Nekvindova, P., Hyps, P., Lyutakov, O., and Jerabek, V., "Flexible polymer planar optical waveguides," Radioengineering 23(3) (2014). 